\title{
Cooperative anti-collision algorithm based on relay sensor in RFID system Xinxian $\mathrm{Li}^{1,}$, , Xiaoling Sun ${ }^{2, \mathrm{~b}}$, Weiqin $\mathrm{Li}^{2, \mathrm{c}}$, Daisong $\mathrm{Shi}^{2, \mathrm{~d}}$ \\ ${ }^{1}$ NanYang Medical College, Nanyang, 473061, China; \\ ${ }^{2}$ Southwest Petroleum University, Chengdu, 610500, China \\ aemail:sollor@126.com, bemail:84598835@qq.com, cemail:nengdee@163.com, ${ }^{\mathrm{d}} 272529426 @ q q . c o m$
}

Keywords: Radio Frequency Identification system; relay sensor; Collaboration; anti-collision

\begin{abstract}
To the RFID system, Multiple reader and transponders deployed to meet the diversity applications, heterogeneous networks, diversity of service application system, lead to the decline of recognition, data transmission delay and signal integrity performance problems. Collaborative anti-collision algorithm suitable for multi-reader and transponder system based on relay sensor is studied. Firstly, proposed an effective scheme of relay sensor structure by combining with the impact of the data and the weight of effect, then, design the suitable peripheral circuits; finally, presents the collaborative anti-collision algorithm based on the relay sensor of the system covers the signal area of three kinds of radiation element convergence. The test results show that the proposed algorithm is superior to the traditional ALOHA algorithm in the read time slot and transmission delay, etc.
\end{abstract}

\section{Introduction}

In the RFID system[1], the important factors affecting the integrity of the data transmission is more than one transponder compete the wireless channel at the same time, then leads to the reader can't confirm the data source, and occur the data collision [2].Thus, in the reader detection range, the multiple transponders need to eliminate other signal interference by identifying the correct signal, and establish the right, stable and reliable communication between the reader and transponder [3], become the key issues in application of RFID system. During the multiple reader session, Fyhn. $\mathrm{K}$ and others [4] improved the reliability of marking probability estimation based on the actual tree anti-collision protocol integration and reliable markers algorithm. The literature [5] combined with the key factor affecting the recognition rate of the RFID system. The Document [6] optimized the search efficiency to reduce the system idle rate. The literature [7] proposed the tire embedded uhf passive RFID system power transmission model.

According to solve the multi class applications, diversity of service application problems of the multiple reader colliding with data in the RFID system, a collaborative anti-collision mechanism based on relay sensor is studied in this paper for a suitable for RFID system. The main work is as follows:(1)Design a relay sensor that can avoid data collision;(2) Design the sensor peripheral circuits in RFID system relay sensor collaboration;(3)Based on the signal of heterogeneous radiation element in system working area, through relay sensor converge, then put forward a collaborative anti-collision algorithm based on.

\section{Relay sensor of the RFID system}

The traditional RFID system including the transponder, reader and diversity of high-level application. For the diversity of complex applications often appear in the data communication between reader and responder. The data collision problems is serious, which results in a decline in the efficiency and accuracy of identification of the transponder.

Assuming that the RFID system, the data collision probability is $\mathrm{Pt}$, which can be divided into the following three kinds of situations:

The data collision between the same transponder and multiple reader, the probability is 
denoted as $P_{c 1}$; The data collision between the same reader and multiple transponder, the probability is denoted as $P_{c 2}$; the data collision between $\mathrm{n}$ readers and $\mathrm{m}$ transponders, the probability is denoted as $P_{c 3}$.As a result, from the perspective of probability distribution, the data collisions in the RFID system is shown in formula (1).

$$
\begin{aligned}
& \left\{\begin{array}{c}
\exp \left(P_{c 1}\right)+\exp \left(P_{c 2}\right)+\exp \left(f\left(P_{c 3}, n, m\right)\right)=P_{t} \\
\quad f\left(P_{c 3}, n, m\right)=\frac{n}{2 m} f\left(P_{c 3}, \frac{n}{m}, \frac{m}{n}\right)
\end{array}\right. \\
& \left\{\begin{array}{l}
\lambda=\widehat{N}_{t} \beta d S \\
S=n m d_{0}
\end{array}\right.
\end{aligned}
$$

Current generation inductively coupled bring electromagnetic state change caused by data collision, the interference to the data communication system, interference weights $\lambda$ can be calculated by the formula (2).Among them, $S$ for collision detection area, $d_{0}$ for effective communication distance, $\beta$ as the magnetic induction intensity, especially the impact of the third data collision on system signal identification and positioning, distribution of the measurement is shown in Fig. 1

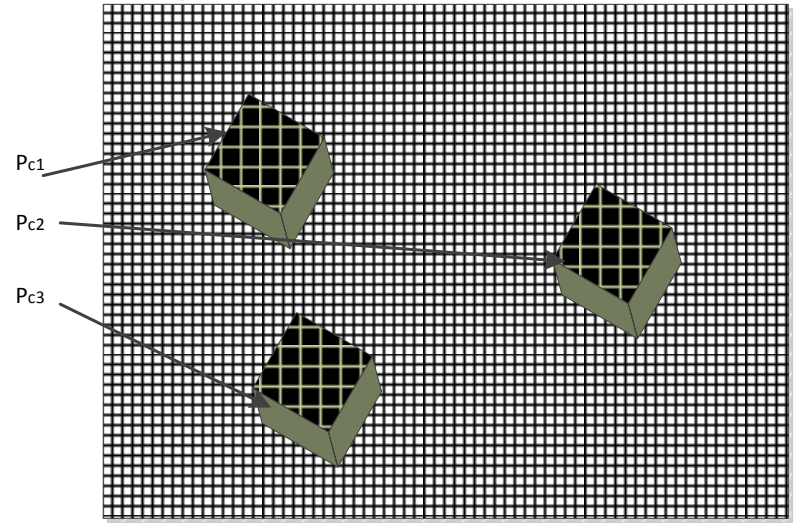

Fig. 1. Distribution of data collision effect

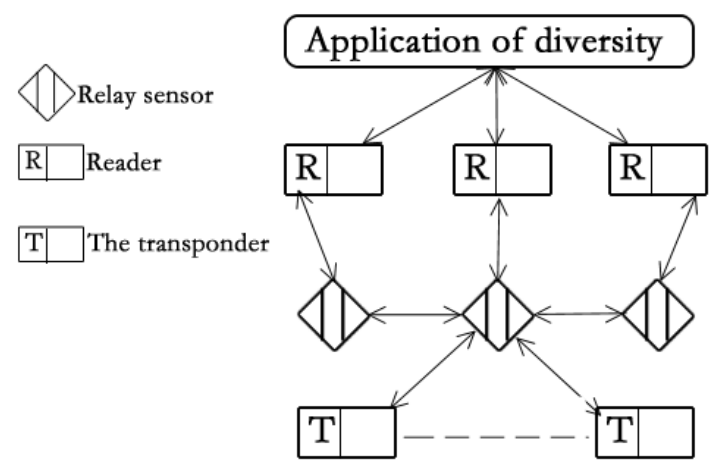

Fig. 2. Collaborative RFID system architecture

In order to eliminate the impact effect as shown in Fig. 2, the traditional RFID system is extended, the structure as shown in Fig. 1 is enhanced as shown in Fig. 3, collaborative RFID system contain collaborative transponder, collaborative reader and relay node. The probability distribution of collision data in Collaborative RFID system as shown in Fig. 2 meets the Poisson properties such as formula (3) have shown, then there is a boundary conditions shows in the formula (4).

$$
\begin{aligned}
& \left\{\begin{array}{c}
\Delta p=-P_{t} \\
f=-P_{t}
\end{array}\right. \\
& \frac{\partial p}{\partial P_{t}}=0
\end{aligned}
$$

where $\mathrm{p}$ is the average data collision probability after the collaborative process. To provide efficient and reliable data recognition and beacon positioning, the function of data collision prevention through a set of relay sensors, the relay sensor must have two parts: the antenna circuit can build a wireless communication with the transponder and reader; the micro controller and the sensor has the collaborative processing function. The head structure of relay sensor as shown in Fig. 3. 


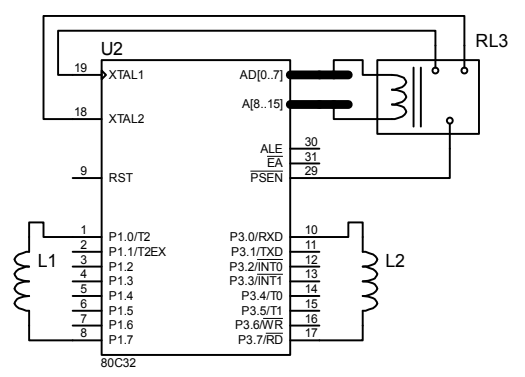

Fig. 3. Structure of relay sensor head

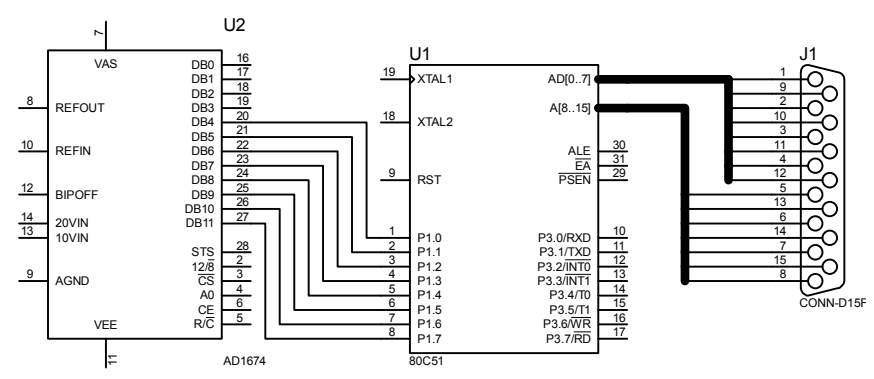

Fig. 4. Structure of relay sensor

Using the Relay sensor design as shown in Fig. 3, through collaborative cooperation between the sensor, reader and transponder, the measurement distribution of data collision effect can be optimized and improved shown in Fig. 2.

\section{Cooperative anti-collision algorithm}

Based on the section design of relay sensor head structure shown in Fig. 3, the Fig. 4 shows the peripheral circuit structure, selection of components include: 16-bit 80C51 microprocessor, CONN-D15F connector, etc. Among them, 15 pin of the connector connected to the microprocessor A0 A14 pin, A16 is idle bit. The radiation element of RFID system with the relay sensor node includes three types: current element, magnetic sensing element and the sensing element. The electromagnetic field space composed by them as shown in formula (5).

$$
\left\{\begin{array}{c}
I=\frac{n}{2 \pi r^{2}} \cos (\omega+\theta) H_{1} \\
\Phi=\frac{3 \pi}{2} \sin \left(\theta^{H_{2}}\right) \\
S=\frac{1}{4 \pi r^{3}} \cos \theta \\
H_{1}=\frac{\omega}{2 \pi} \\
H_{2}=\frac{3 \pi}{4} \sin \theta
\end{array}\right.
$$

Where $I$ is the current element, $\Phi$ is magnetic element and $S$ is the sensing element for signal strength, $\mathrm{n}$ represents the number of turns of the coil, $\omega$ is system operating frequency, $\theta$ is the angle radian of the relay sensor and antenna coil origin, ${ }^{H_{1}}$ represents the coefficient that the magnetic field strength influence the current, $\mathrm{H}_{2}$ represents the coefficient that perception signal of relay sensor influence the magnetic.

In the electromagnetic field covered by the radiation of the three elements, heterogeneous signal transmission cycle process as shown in Fig. 5.The power on process is passive transponder through magnetic induction coupling voltage from the reader, and the process of relay sensor batteries supply power for microprocessor and front-end data launch process. The initialization work includes: reader sent detection signal to find all transponder in the function area, transponder reply to reader response and relay sensor interference the collision perception of multiple transponder and reader. The specific signals include transponder, relay and summary signals.

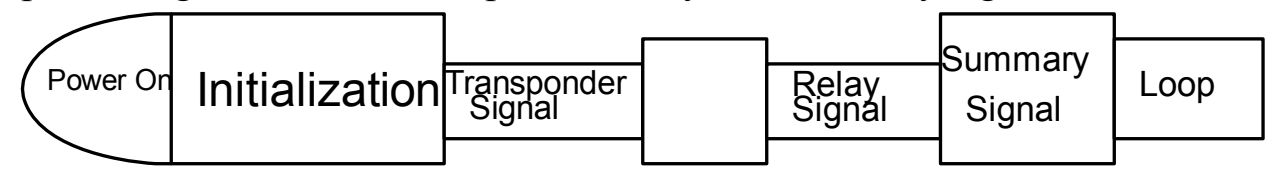

Fig. 5. Relay sensor and transponder terminal voltage loop transmission

The cooperative Anti-collision algorithm based on relay sensor process as follows :

(1) Multiple reader, multiple transponders and relay sensor power on;

(2) Initializes the reader and the transponder chip, microprocessor and peripheral circuit of relay 
sensor;

(3) Multiple reader request command frame and the response of the transponder command frame converge to relay sensor nodes;

(4) Collaboration between the relay sensor nodes, using formula (1) to (3) get the cooperative gain;

(5) According to the formula (4) and (5) get the system summary signal;

(6) According to the formula (5), the reader obtained the correctly transponder response data send from the relay sensor after the anti-collision .

\section{Test}

In order to contrast and analyze the performance of the anti-collision algorithm and the ALONA algorithm in RFID system without the relay sensor, the experimental environment layout is as follows:

(1)Deploy 10 similar application reader in the held area of $100 * 80$ meters;

(2)Deploy 50 similar application transponders;

(3)Deploy 5 similar relay sensors, and its parameters are shown in table.1.

Table.1. Parameter setting of relay sensor

\begin{tabular}{|c|c|c|c|}
\hline parameters & value & parameters & value \\
\hline The output current & $10-20 \mathrm{~mA}$ & emissivity & $0.1-1.1$ \\
\hline Working distance & $10-20 \mathrm{~m}$ & load resistance & $>50 \mathrm{k} \Omega$ \\
\hline
\end{tabular}

In first set of experiments, set response frame number of the transponder reply to reader from the beginning of 10, increase to 50 with a step of 10 , count and measure the number of reading time slots of the reader through relay sensor, the result shown in Fig. 6. It is turned out that the traditional ALOHA anti-collision algorithm with the increase of the transponder number in the system, the reader read the required time slots number increases rapidly. However, the proposed algorithm for the transponder joined the relay sensor between readers which reduces the system resources of anti-collision occupies, effectively reduces the required read time slots.

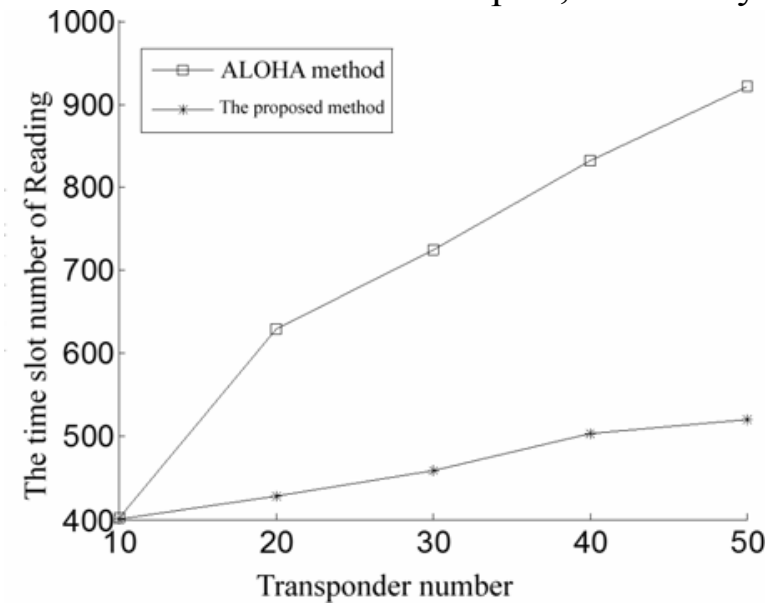

Fig. 6 Read time slot number

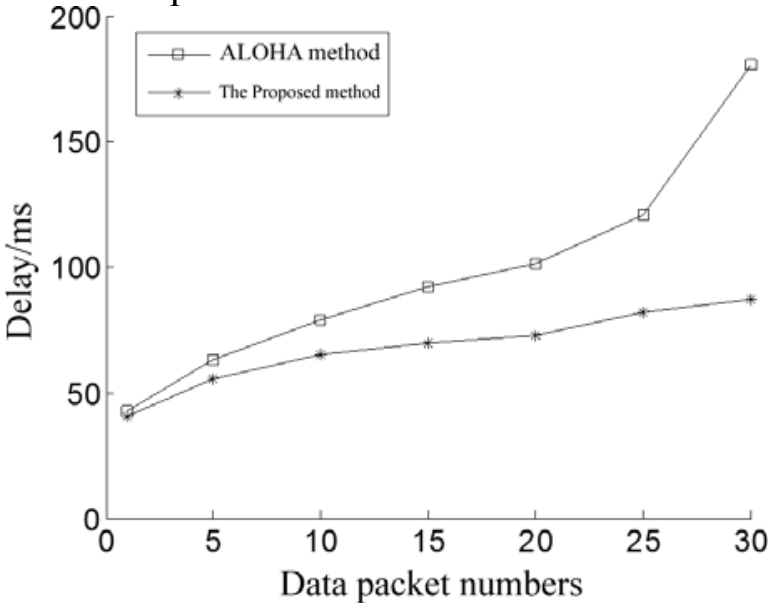

Fig. 7 Transmission Delay

In the second set of experiments, control the data frame number of RFID system from the beginning of 1 , increase to 30 with a step of 5 , count and measure transmission delay from the transponder to the reader, the result shown in Fig. 7. It is turned out that the two anti-collision algorithms in the scale of the data frame increases from 1 to 15 , the delay changed in same trend, the delay of proposed algorithm significantly shorter than ALOHA algorithm. Especially when the number of data frames to achieve 20, the delay of the traditional ALOHA algorithm suddenly increased rapidly, but the proposed algorithm remains stable trend, this is mainly due to the relay sensor collaborative anti-collision algorithm, through the summary of signal from the reader, transponder and relay sensor, arrive to the reader or transponder after correctly collaborative process by relay sensor, provides a reliable guarantee for its communication. 


\section{Conclusion}

The collaborative anti-collision algorithm based on relay sensor is studied in this paper for a suitable for multi reader transponder system. Firstly, according to the three situations occur collision data system, combined with the data of impact angle and impact effect of weight, put forward an effective avoid the data collision design scheme of relay sensor structure; then, design the suitable peripheral circuits in RFID system relay sensor collaboration; finally, the system covers the signal area of three kinds of radiation element convergence based on the relay sensor and presents a collaborative anti-collision algorithm based on. Experimental results show that in the read time slot and the transmission delay, the proposed ALOHA algorithm for collaborative anti-collision algorithm is obviously superior to the traditional.

\section{Acknowledgement}

In this paper, the research was sponsored by the Nature Science Foundation of Henan Province (Project No. 20530973106791).

\section{References}

[1] Cantina, Yu-ping Zhao, Timor Korhonen, RFID system research collision agreement - design and optimization [J]. Journal of electronics and information. 2012, 31 (1): 1-4.

[2] Xie Shengmei, Jun-hui Zhao. Based on the principle of optimization in the RFID system using collision algorithm study [J]. Journal of circuits and systems, 2014, 14 (4): 8-12.

[3] Tong Weiqin. Service oriented in the RFID system dynamic resource allocation optimization mechanism [J]. Journal of communication. 2012, 33 (8): 93-105.

[4] Fyhn k., Jacobsen, R.M. Popovski p. Fast Capture Recapture Approach for Mitigating the Problem of Missing the RFID Tags [J]. IEEE the Transactions on Mobile Computing, 2012, 11 (3): 518-528.

[5] Ya-li liu, Sheng-bo Hu, Yan-fu Yu. Embedded RFID system identification key factor analysis [J]. Journal of engineering design. 2015, 19 (6): 479-484.

[6] Guo Rongzuo, Zhang, Huangjun. RFID system more than the 8-4-2 dynamic octree collision algorithm design and analysis [J]. Journal of Chong Qing normal university (natural science edition) journal. 2013, (06): 118-122.

[7] Sheng-bo Hu, soldiers, Shu Heng. Tire embedded power transfer characteristic of uhf passive RFID system [J]. Journal of agricultural engineering. 2013, 29 (21): 150-158. 